\title{
The Robustness of LWPP and WPP, with an Application to Graph Reconstruction
}

\author{
Edith Hemaspaandra
}

Rochester Institute of Technology, Rochester, NY, USA

Lane A. Hemaspaandra

University of Rochester, Rochester, NY, USA

Holger Spakowski

University of Cape Town, Rondebosch, South Africa

Osamu Watanabe

Tokyo Institute of Technology, Tokyo, Japan

\begin{abstract}
We show that the counting class LWPP [8] remains unchanged even if one allows a polynomial number of gap values rather than one. On the other hand, we show that it is impossible to improve this from polynomially many gap values to a superpolynomial number of gap values by relativizable proof techniques.

The first of these results implies that the Legitimate Deck Problem (from the study of graph reconstruction) is in LWPP (and thus low for PP, i.e., $\mathrm{PP}^{\text {Legitimate Deck }}=\mathrm{PP}$ ) if the weakened version of the Reconstruction Conjecture holds in which the number of nonisomorphic preimages is assumed merely to be polynomially bounded. This strengthens the 1992 result of Köbler, Schöning, and Torán [15] that the Legitimate Deck Problem is in LWPP if the Reconstruction Conjecture holds, and provides strengthened evidence that the Legitimate Deck Problem is not NP-hard.

We additionally show on the one hand that our main LWPP robustness result also holds for WPP, and also holds even when one allows both the rejection- and acceptance- gap-value targets to simultaneously be polynomial-sized lists; yet on the other hand, we show that for the \#P-based analog of LWPP the behavior much differs in that, in some relativized worlds, even two target values already yield a richer class than one value does.
\end{abstract}

2012 ACM Subject Classification Theory of computation $\rightarrow$ Complexity classes

Keywords and phrases structural complexity theory, robustness of counting classes, the legitimate deck problem, PP-lowness, the Reconstruction Conjecture

Digital Object Identifier 10.4230/LIPIcs.MFCS.2018.51

Related Version A full version of the paper can be found at [12], https://arxiv.org/abs/ 1711.01250v2.

\section{Introduction}

Nothing is more natural than wanting to better understand an object by knowing what it can and cannot do. Whether wondering how fast a (rental?) car can go in reverse or wondering if $\mathrm{NP}^{\mathrm{NP}}$ can without loss of generality be assumed to ask at most one question per nondeterministic path (as it indeed can, as is implicit in the quantifier characterization [27, 30] of $\mathrm{NP}^{\mathrm{NP}}$ ), we both in life and as theoreticians want to find how robust things are.

(c) (i) Edith Hemaspaandra, Lane A. Hemaspaandra, Holger Spakowski, and Osamu Watanabe;

43rd International Symposium on Mathematical Foundations of Computer Science (MFCS 2018).

Editors: Igor Potapov, Paul Spirakis, and James Worrell; Article No. 51; pp. 51:1-51:14

Leibniz International Proceedings in Informatics

LIPICS Schloss Dagstuhl - Leibniz-Zentrum für Informatik, Dagstuhl Publishing, Germany 
We are often particularly happy when a class proves to be quite robust under definitional perturbations. Such robustness on one hand suggests that perhaps there is something broadly natural about the class, and on the other hand such robustness often makes it easier to put the class to use.

This paper shows that the counting classes LWPP and WPP, defined in 1994 in the seminal work of Fenner, Fortnow, and Kurtz [8] on gap-based counting classes, are quite robust. Even though their definitions are in terms of having the gap function (the difference between the number of accepting and rejecting paths of a machine) hit a single target value, we prove (in Section 3) that one can allow a list of up to polynomially many target values without altering the descriptive richness of the class, i.e., without changing the class.

We then apply this to the question of whether the Legitimate Deck Problem is in LWPP.

The Legitimate Deck Problem (a formal definition will be given in Section 4) is the decision problem of determining whether, given a multiset of (unlabeled) graphs, there exists a graph $G$ such that that multiset is precisely (give or take isomorphisms) the multiset of one-nodedeleted subgraphs of $G$ (a.k.a. the deck of $G$ ) [17]. The Reconstruction Conjecture [13, 28] - which in the wake of the resolution of the Four-Color Conjecture was declared by the editorial board of the Journal of Graph Theory to be the foremost open problem in graph theory [7] - states that every graph with three or more nodes is uniquely determined (give or take isomorphisms) by its multiset of one-node-deleted subgraphs. The Legitimate Deck Problem was defined in 1978 by Nash-Williams [22], in his paper that framed the algorithmic/complexity issues related to reconstructing graphs - such as telling whether a given deck is legitimate (i.e., is the deck of some graph).

Our application of our LWPP robustness result to the question of whether the Legitimate Deck Problem is in LWPP is the following. The strongest previous evidence of the simplicity of the Legitimate Deck Problem is the 1992 result of Köbler, Schöning, and Torán [15] that the Legitimate Deck Problem is in LWPP (and thus is PP-low, i.e., PPLegitimate Deck $=$ PP) if the Reconstruction Conjecture (i.e., that each deck whose elements all have at least two nodes has at most one preimage, give or take isomorphisms) holds. Using this paper's main robustness result as a tool, Section 4 proves that the Legitimate Deck Problem is in LWPP (and thus is PP-low) if a weakened version of the Reconstruction Conjecture holds, namely, that each deck has at most a polynomial number of nonisomorphic preimages.

This weakened version is not known to be equivalent to the Reconstruction Conjecture itself. And so our result for the first time gives a path to proving that the Legitimate Deck Problem is PP-low that does not require one to, on the way, resolve the foremost open problem in graph theory [7].

We started this section by noting that it is natural to want to know both flexibilities and limitations of classes. Our main result is about flexibility: going from one target gap to instead a polynomial number. But are we leaving money on the table? Could we extend our result to slightly superpolynomial numbers of target gaps, or even to exponential numbers of target gaps? In Section 5 we note that if the robustness of LWPP were to hold up to exponentially many target gaps, then NP would be in LWPP and so would be PP-low (i.e., $\mathrm{PP}^{\mathrm{NP}}=\mathrm{NP}$ ) ; yet $\mathrm{NP}$ is widely suspected not to be PP-low. We also, by encoding nondeterministic oracle Turing machines by low-degree multivariate polynomials so as to capture the gap functions of those machines, show an oracle relative to which robustness fails for all superpolynomial numbers of target gaps; thus, no extension beyond this paper's polynomial-number-of-target-gaps robustness result for LWPP can be proven by a relativizable proof. And for the \#P-based analogue of LWPP, in Section 6 we show that even allowing two target values yields, in some relativized worlds, a richer class than one 
target value. We also (in the final part of Section 3) extend our main result to show that the simultaneous expansion to polynomial-sized lists of both the acceptance and rejection target-gap lists still, for LWPP and WPP, yields the classes LWPP and WPP.

To summarize: In this paper, we prove that LWPP and WPP are robust enough that they remain unchanged when their single target gap is allowed to be expanded to a polynomial-sized list; we apply this new robustness of LWPP to show that the PP-lowness of the Legitimate Deck Problem follows from a weaker hypothesis than was previously known; we show that our polynomial robustness of LWPP is optimal with respect to relativizable proofs; and we prove a number of related results on limitations and extensions.

\section{Preliminaries}

We first present the definitions of many of the counting classes that we will be speaking of, taking the definitions from the seminal paper of Fenner, Fortnow, and Kurtz [8].

Definition 2.1 ([8]).

1. For each nondeterministic polynomial-time Turing machine $N$, the function $\operatorname{acc}_{N}: \Sigma^{*} \rightarrow \mathbb{N}$ is defined such that for every $x \in \Sigma^{*}, \operatorname{acc}_{N}(x)$ equals the number of accepting computation paths of $N$ on input $x$.

2. For each nondeterministic polynomial-time Turing machine $N$, the function $\operatorname{rej}_{N}: \Sigma^{*} \rightarrow \mathbb{N}$ is defined such that for every $x \in \Sigma^{*}$, $\operatorname{rej}_{N}(x)$ equals the number of rejecting computation paths of $N$ on input $x$.

3. For each nondeterministic polynomial-time Turing machine $N$, the function $\operatorname{gap}_{N}: \Sigma^{*} \rightarrow$ $\mathbb{Z}$ is defined such that for every $x \in \Sigma^{*}$,

$$
\operatorname{gap}_{N}(x)=\operatorname{acc}_{N}(x)-\operatorname{rej}_{N}(x) .
$$

- Definition 2.2 ([8]).

GapP $=\left\{\operatorname{gap}_{N} \mid N\right.$ is a polynomial-time nondeterministic Turing machine $\}$.

- Definition $2.3([23,8])$. SPP is the class of all sets $A$ such that there exists a GapP function $g$ such that for all $x \in \Sigma^{*}$,

$$
\begin{aligned}
& x \in A \Longrightarrow g(x)=1 \\
& x \notin A \Longrightarrow g(x)=0 .
\end{aligned}
$$

The following class, WPP, is potentially larger than SPP. Instead of the "target value" 1 for the case $x \in A$, we allow a target value $f(x)$, where $f$ may be any polynomial-time computable function whose image does not contain 0 . FP denotes the class of polynomial-time computable functions.

- Definition 2.4 ([8]). WPP is the class of all sets $A$ such that there exists a GapP function $g$ and a function $f \in \mathrm{FP}$ that maps from $\Sigma^{*}$ to $\mathbb{Z}-\{0\}$ such that for all $x \in \Sigma^{*}$,

$$
\begin{aligned}
& x \in A \Longrightarrow g(x)=f(x) \\
& x \notin A \Longrightarrow g(x)=0 .
\end{aligned}
$$

The class LWPP is the same as WPP except that the "target function" $f$ may depend on only the length of the input. 
Definition 2.5 ([8]). LWPP is the class of all sets $A$ such that there exists a GapP function $g$ and a function $f \in \mathrm{FP}$ that maps from $0^{*}$ to $\mathbb{Z}-\{0\}$ such that for all $x \in \Sigma^{*}$,

$$
\begin{aligned}
& x \in A \Longrightarrow g(x)=f\left(0^{|x|}\right) \\
& x \notin A \Longrightarrow g(x)=0 .
\end{aligned}
$$

Here, as usual, for any $x \in \Sigma^{*},|x|$ denotes the length of $x$, and for any $n \in \mathbb{N}$, $0^{n}$ is the string consisting of exactly $n$ zeroes.

We now generalize the definition of LWPP to the case of having the target of the GapP function be, for members of the set, not a single value but a collection of values.

One might expect us to formalize this by simply having the polynomial-time computable "what is the target" function output a list of the nonzero-integer targets. That would work fine and be equivalent to what we are about to do, as long as we are dealing with lists having at most a polynomial number of elements. However, to be able to speak of even longer lists - as will be important in our negative results offsetting our main result - we use an indexing approach, as follows.

- Definition 2.6. Let $r$ be any function mapping from $\mathbb{N}$ to $\mathbb{N}$. Then the class $r$-LWPP is the class of all sets $A$ such that there exists a GapP function $g$ and a function $f \in$ FP that maps to $\mathbb{Z}-\{0\}$ such that for each $x \in \Sigma^{*}$,

$$
\begin{aligned}
& x \in A \Longrightarrow \text { there exists } i \in\{1,2, \ldots, r(|x|)\} \text { such that } g(x)=f\left(\left\langle 0^{|x|}, i\right\rangle\right) \\
& x \notin A \Longrightarrow g(x)=0 .
\end{aligned}
$$

The following class, Poly-LWPP, will be central to this paper: Our main result is that this class in fact equals LWPP. The " $+c$ " in Definition 2.7 may seem strange at first. But without it we would have a boundary-case pathology at $n=0$, namely, the class could not contain any set that contains the empty string. ${ }^{1}$

\section{- Definition 2.7.}

$$
\text { Poly-LWPP }=\bigcup_{c \in \mathbb{N}^{+}}\left(n^{c}+c\right) \text {-LWPP. }
$$

It is easy to see that 1-LWPP $=$ LWPP, and that, of course, more flexibility as to targets never removes sets from the class, i.e., speaking loosely for the moment as to notation (and the $\log$ case will not be defined or used again in this paper, but it is clear from context here what we mean by it; the exponential case's definition can be found in the full version of the paper [12]), 1 -LWPP $\subseteq 2$-LWPP $\subseteq 3$-LWPP $\subseteq \cdots \subseteq$ Log-LWPP $\subseteq$ Poly-LWPP $\subseteq$ Exp-LWPP. As mentioned above, in this paper we will prove that the first five of these "ᄃ"s are in fact all equalities. We will also prove that the sixth " $\subseteq$ " cannot be an equality unless NP is PP-low.

We now show that for every function $r, r$-LWPP is contained in the co-class of the well-known counting class $\mathrm{C}_{=} \mathrm{P}$.

1 The " $+c$ " in Definition 2.7 also, on its surface, would seem to make a difference at length 1 , by allowing lists of size greater than one; however, one could work around that issue. In contrast, the exclusion of the empty string would not be avoidable if our class of polynomials were to be a class - such as $n^{c}-$ such that all of its members evaluate to 0 at $n=0$. In any case, our use of $n^{c}+c$ avoids any special worries at lengths 0 and 1 . And since for every polynomial $p$ there is a $c$ such that $(\forall n \in \mathbb{N})\left[p(n) \leq n^{c}+c\right]$, using polynomials just of the form $n^{c}+c$ is in fact not a restriction. 
- Definition $2.8([25,29]) . \mathrm{C}_{=} \mathrm{P}$ is the class of all sets $A$ such that there is a nondeterministic polynomial-time Turing machine $N$ and a function $f \in \mathrm{FP}$ such that for each $x \in \Sigma^{*}$,

$x \in A \Longleftrightarrow \operatorname{acc}_{N}(x)=f(x)$.

More convenient for us is the following characterization of $\mathrm{C}_{=} \mathrm{P}$ using GapP functions.

- Theorem 2.9 ([8]). For each $A \subseteq \Sigma^{*}, A \in \mathrm{C}_{=} \mathrm{P}$ if and only if there exists a function $g \in \mathrm{GapP}$ such that for all $x \in \Sigma^{*}$,

$x \in A \Longleftrightarrow g(x)=0$.

We thus certainly have the following, which holds simply by taking the GapP function $g$ required in Theorem 2.9 to be the same as the function $g$ in Definition 2.6.

Theorem 2.10. For each function $r: \mathbb{N} \rightarrow \mathbb{N}, r$-LWPP $\subseteq \mathrm{coC}_{=} \mathrm{P}$.

\section{Main Result: LWPP Stays the Same If for Accepted Inputs We Allow Polynomially Many Gap Values Instead of One}

We now state our main result: LWPP altered to allow even a polynomial number of target gap values is still LWPP (i.e., with just one target gap value).

Theorem 3.1. Poly-LWPP $=$ LWPP.

For the proof, we need the following closure properties shown by Fenner, Fortnow, and Kurtz $[8] .^{2}$ For function classes $\mathcal{F}_{1}$ and $\mathcal{F}_{2}, \mathcal{F}_{1} \circ \mathcal{F}_{2}=\left\{f_{1} \circ f_{2} \mid f_{1} \in \mathcal{F}_{1} \wedge f_{2} \in \mathcal{F}_{2}\right\}$, where $\circ$ denotes composition.

- Closure Property 3.2 ([8]). GapP $\circ \mathrm{FP}=\mathrm{GapP}$ and $\mathrm{FP} \subseteq$ GapP.

- Closure Property 3.3 ([8]). If $g \in \mathrm{GapP}$ then $-g \in \mathrm{GapP}$.

- Closure Property 3.4 ([8]). If $g \in \mathrm{GapP}$ and $q$ is a polynomial, then the function

$$
h(x)=\prod_{0 \leq i \leq q(|x|)} g(\langle x, i\rangle)
$$

is in GapP.

- Closure Property 3.5 ([8]). GapP is closed under addition, subtraction, and multiplication.

Proof of Theorem 3.1. As mentioned previously, it is easy to see that LWPP $\subseteq$ Poly-LWPP.

To show Poly-LWPP $\subseteq$ LWPP, let $A$ be a set in Poly-LWPP defined by $g \in$ GapP, $f \in \mathrm{FP}$, and polynomial $r(n)=n^{c}+c$ according to Definitions 2.6 and 2.7.

Let $h_{1}$ be a function such that for all $x \in \Sigma^{*}$ and $i \in \mathbb{N}^{+}$,

$$
h_{1}(\langle x, i\rangle)=f\left(\left\langle 0^{|x|}, i\right\rangle\right)-g(x) .
$$

\footnotetext{
2 We mention in passing that, regarding Closure Property 3.4, if the polynomial $q$ were allowed to have coefficients that are uncomputable - or that are extremely expensive to compute prefixes of the values of - real numbers, that claimed closure property might fail; we here are, as is typical in such settings, tacitly assuming that the polynomials have rational coefficients.
} 
We have $h_{1} \in \mathrm{GapP}$ since $f \in \mathrm{FP} \subseteq \mathrm{GapP}, g \in \mathrm{GapP}$, and GapP is closed under subtraction [8]. We define $h_{2}$ such that for all $x \in \Sigma^{*}$,

$$
h_{2}(x)=\prod_{1 \leq i \leq r(|x|)} h_{1}(\langle x, i\rangle) .
$$

By Closure Property $3.4, h_{2} \in$ GapP. Note that for all $x \in \Sigma^{*}$,

$$
h_{2}(x)=\prod_{1 \leq i \leq r(|x|)}\left(f\left(\left\langle 0^{|x|}, i\right\rangle\right)-g(x)\right) .
$$

It follows that for every $x \in \Sigma^{*}$,

$$
h_{2}(x)=\left\{\begin{array}{lc}
0 & \text { if there exists } i \in\{1,2, \ldots, r(|x|)\} \\
\text { such that } g(x)=f\left(\left\langle 0^{|x|}, i\right\rangle\right) \\
\prod_{1 \leq i \leq r(|x|)} f\left(\left\langle 0^{|x|}, i\right\rangle\right) & \text { if } g(x)=0 .
\end{array}\right.
$$

Now we define the function $\widehat{g}$ such that for all $x \in \Sigma^{*}$,

$$
\widehat{g}(x)=h_{2}(x)-\prod_{1 \leq i \leq r(|x|)} f\left(\left\langle 0^{|x|}, i\right\rangle\right) .
$$

Using the closure properties, it is easy to see that $\widehat{g} \in \mathrm{GapP}$.

Note that by Eqn. (1), we have that for all $x \in \Sigma^{*}$,

$$
\widehat{g}(x)= \begin{cases}-\prod_{1 \leq i \leq r(|x|)} f\left(\left\langle 0^{|x|}, i\right\rangle\right) & \text { if there exists } i \in\{1,2, \ldots, r(|x|)\} \\ 0 & \text { such that } g(x)=f\left(\left\langle 0^{|x|}, i\right\rangle\right) \\ & \text { if } g(x)=0 .\end{cases}
$$

Let $\widehat{f}$ be a function such that for all $\ell \in \mathbb{N}$,

$$
\widehat{f}\left(0^{\ell}\right)=-\prod_{1 \leq i \leq r(\ell)} f\left(\left\langle 0^{\ell}, i\right\rangle\right) .
$$

It is easy to see that $\widehat{f} \in \mathrm{FP}$. Keeping in mind Eqn. (2), it follows from the above that for every $x \in \Sigma^{*}$,

$$
\widehat{g}(x)= \begin{cases}\widehat{f}\left(0^{|x|}\right) & \text { if } x \in A \\ 0 & \text { otherwise }\end{cases}
$$

Since $\widehat{g} \in \mathrm{GapP}$ and $\widehat{f} \in \mathrm{FP}$, this implies that $A \in \mathrm{LWPP}$.

A theorem analogous to Theorem 3.1 also holds for the corresponding class that allows the target values to depend on the actual input instead on only the length of the input.

\section{- Definition 3.6.}

$$
\text { Poly-WPP }=\bigcup_{c \in \mathbb{N}^{+}}\left(n^{c}+c\right)-\mathrm{WPP} .
$$

- Theorem 3.7. Poly-WPP $=\mathrm{WPP}$. 
The proof is almost exactly the same as the proof of Theorem 3.1, simply taking into account the fact that for WPP the "gap" function can vary even among inputs of the same length.

We remark that the robustness results stated in Theorems 3.1 and 3.7 do not seem to in any obvious way follow as corollaries to the closure of LWPP under polynomial-time Turing reductions [8] or of WPP under polynomial-time truth-table reductions [26].

Let us now see whether we can extend Theorems 3.1 and 3.7. Suppose we not only allow the target-gap set for acceptance to have polynomially many values, but in addition allow the target-gap set for rejection to have polynomially many values. (In contrast, both LWPP and $r$-LWPP allow rejection only when the gap's value is 0$)$. Is LWPP so robust that even that class is no larger than LWPP? We will now build on our main result to give the answer "yes" to that question, thus extending our main result to this more symmetric case for LWPP and for WPP.

To this end, let us define $\left(r_{A}, r_{R}\right)$-LWPP as follows. (One of course can in the clear, analogous way define a similarly loosened version of WPP, $\left(r_{A}, r_{R}\right)$-WPP.)

- Definition 3.8. Let $r_{A}$ and $r_{R}$ be any functions mapping from $\mathbb{N}$ to $\mathbb{N}$. Then $\left(r_{A}, r_{R}\right)$-LWPP is the class of all sets $B$ such that there exists a GapP function $g$ and functions - each mapping to $\mathbb{Z}-f_{A} \in \mathrm{FP}$ and $f_{R} \in \mathrm{FP}$ such that both of the following hold:

1. For each $j \in \mathbb{N}, A_{j} \cap R_{j}=\emptyset$, where

$$
A_{j}=\left\{n \mid\left(\exists i \in\left\{1,2, \ldots, r_{A}(j)\right\}\right)\left[f_{A}\left(\left\langle 0^{j}, i\right\rangle\right)=n\right]\right\}
$$

and

$$
R_{j}=\left\{n \mid\left(\exists i \in\left\{1,2, \ldots, r_{R}(j)\right\}\right)\left[f_{R}\left(\left\langle 0^{j}, i\right\rangle\right)=n\right]\right\} .
$$

2. For each $x \in \Sigma^{*}$,

$$
\begin{aligned}
& x \in B \Longrightarrow g(x) \in A_{|x|} \\
& x \notin B \Longrightarrow g(x) \in R_{|x|} .
\end{aligned}
$$

It is not hard to see, via shifting gaps with dummy paths, that the class $(r, 1)$-LWPP equals $r$-LWPP. However, what can be shown more generally about the classes $\left(r_{A}, r_{R}\right)$-LWPP? For example, for which functions $r_{1, A}, r_{1, R}, r_{2, A}$, and $r_{2, A}$ does it hold that $\left(r_{1, A}, r_{1, R}\right)$-LWPP $\subseteq$ $\left(r_{2, A}, r_{2, R}\right)$-LWPP? (There are some literature notions that, in different ways, have at least a somewhat similar flavor to our notion, namely, defining classes by being more flexible regarding acceptance types. In particular, the counting classes $\mathrm{CP}_{S}$ of Cai et al. [4] and the "C-class" framework of Bovet, Crescenzi, and Silvestri [3] have such a flavor. But in contrast with those, our classes here are ones whose definitions are centered on the notion of gaps.) We do not here undertake that general study, but instead resolve what seems the most compelling question, namely, we prove that (Poly, Poly $)-\mathrm{LWPP}=\mathrm{LWPP}$.

Definition 3.9. (Poly, Poly)-LWPP $=\bigcup_{c \in \mathbb{N}^{+}}\left(n^{c}+c, n^{c}+c\right)$-LWPP.

- Theorem 3.10. (Poly, Poly)-LWPP = Poly-LWPP.

The proof of Theorem 3.10 can be found in the full version of the paper [12]. Together with Theorem 3.1, we get the following corollary.

- Corollary 3.11. (Poly, Poly)-LWPP $=$ LWPP.

An analogous statement can also be shown for the analogously defined class (Poly, Poly)WPP:

Theorem 3.12. (Poly, Poly)-WPP $=\mathrm{WPP}$. 


\section{Applying the Main Result to Graph Reconstruction}

- Definition 4.1. Let $\left\langle G_{1}, G_{2}, \ldots, G_{n}\right\rangle$ be a sequence of graphs and $G=(V, E)$ a graph with $V=\{1,2, \ldots, n\}$. Suppose that there is a permutation $\pi \in S_{n}$ such that for each $k \in\{1,2, \ldots, n\}$, the graph $G_{\pi(k)}$ is isomorphic to the graph $(V-\{k\}, E-\{\{k, \ell\}: \ell \in V\})$ obtained by deleting vertex $k$ from $G$. Then $\left\langle G_{1}, G_{2}, \ldots, G_{n}\right\rangle$ is called a deck of $G$ and $G$ is called a preimage of the sequence $\left\langle G_{1}, G_{2}, \ldots, G_{n}\right\rangle$.

A sequence of graphs $\left\langle G_{1}, G_{2}, \ldots, G_{n}\right\rangle$ is called a legitimate deck if there exists a graph $G$ that is a preimage of $\left\langle G_{1}, G_{2}, \ldots, G_{n}\right\rangle$.

The Reconstruction Conjecture (see, e.g., the surveys [2, 21, 1] and the book [18]) says that each legitimate deck consisting of graphs with at least two vertices has exactly one preimage up to isomorphism. This conjecture is a very prominent conjecture in graph theory - as mentioned in Section 1 it is perhaps the most important conjecture in that area - and has been studied for many decades.

Nash-Williams [22], Mansfield [20], Kratsch and Hemachandra [17], and Hemaspaandra et al. [11] introduced various decision problems related to the Reconstruction Conjecture, as part of a stream of work studying the algorithmic and complexity issues of reconstruction. We here are interested mainly in the Legitimate Deck Problem, which is defined as the following decision problem.

Legitimate Deck (a.k.a. the Legitimate Deck Problem) [20]: Given a sequence of graphs $\left\langle G_{1}, G_{2}, \ldots, G_{n}\right\rangle$, is $\left\langle G_{1}, G_{2}, \ldots, G_{n}\right\rangle$ a legitimate deck?

Mansfield [20] showed that the Graph Isomorphism Problem, GI (given two graphs $G_{1}$ and $G_{2}$, are they isomorphic?), is polynomial-time many-one reducible to the Legitimate Deck Problem. However, to this day it remains open whether there is a polynomial-time many-one reduction from the Legitimate Deck Problem to GI.

So how hard is the Legitimate Deck Problem? It is easy to see that the Legitimate Deck Problem is in NP. In the following, we will see that there is some evidence that the Legitimate Deck Problem is not NP-hard, and we will improve that evidence.

Let us define the following function problem.

Preimage Counting [16, 17]: Given a sequence of graphs $\left\langle G_{1}, G_{2}, \ldots, G_{n}\right\rangle$, compute the number PCount $\left(\left\langle G_{1}, G_{2}, \ldots, G_{n}\right\rangle\right)$ of all nonisomorphic preimages for the sequence $\left\langle G_{1}, G_{2}, \ldots, G_{n}\right\rangle$.

Köbler, Schöning, and Torán [15] showed the following theorem.

- Theorem 4.2 ([15]). There is a function $h: 0^{*} \rightarrow \mathbb{N}-\{0\}$ in $\mathrm{FP}$ such that the function that maps every sequence $\left\langle G_{1}, G_{2}, \ldots, G_{n}\right\rangle$ to $h\left(0^{n}\right)$ times the number of nonisomorphic preimages, i.e.,

$$
\left\langle G_{1}, G_{2}, \ldots, G_{n}\right\rangle \mapsto h\left(0^{n}\right) \cdot \operatorname{PCount}\left(\left\langle G_{1}, G_{2}, \ldots, G_{n}\right\rangle\right)
$$

is in GapP.

Theorem 4.2 has the following corollary.

- Corollary 4.3 ([15]). If the Reconstruction Conjecture holds, then the Legitimate Deck Problem is in LWPP. 
Since all LWPP sets are PP-low, that immediately gives some evidence that the Legitimate Deck Problem is not NP-hard.

- Corollary 4.4. If the Reconstruction Conjecture holds, then the Legitimate Deck Problem is not NP-hard (or even NP-Turing-hard) unless NP is PP-low.

Unfortunately, we do not know whether the Reconstruction Conjecture holds. However, perhaps we can prove the membership of the Legitimate Deck Problem in LWPP under a weaker assumption than the Reconstruction Conjecture, for instance, what if the number of nonisomorphic preimages is not always 0 or 1 (as holds under the Reconstruction Conjecture), but rather is merely relatively small, e.g., some constant or some polynomial in the number of vertices (as must hold for each graph class having bounded minimum degree)? We will now use the results of the previous section to prove that this indeed is the case.

- Conjecture 4.5 ( $q$-Reconstruction Conjecture). For each legitimate deck there exist at most $q(n)$ nonisomorphic preimages, where $n$ is the number of graphs in the deck.

For any function $r$, we now define a complexity class $r$ - $\widehat{\mathrm{LWPP}}$. This class may not seem very natural, but we will see that it is very-well suited to helping us classify the problem Legitimate Deck. In some sense, it is a tool that we will use in our proof, and then will discard by noting that it in fact turns out to be a disguised version of $r$-LWPP.

- Definition 4.6. Let $r$ be any function mapping from $\mathbb{N}$ to $\mathbb{N}$. Then the class $r$-LWPP is the class of all sets $A$ such that there exists a GapP function $g$, and a function $f \in \mathrm{FP}$ that maps from $0^{*}$ to $\mathbb{Z}-\{0\}$, such that for each $x \in \Sigma^{*}$,

$x \in A \Longrightarrow$ there exists $i \in\{1,2, \ldots, r(|x|)\}$ such that $g(x)=i \cdot f\left(0^{|x|}\right)$

$x \notin A \Longrightarrow g(x)=0$.

- Theorem 4.7. Let $q$ be any nondecreasing function from $\mathbb{N}$ to $\mathbb{N}$. Then the following holds. If the q-Reconstruction Conjecture holds, then the Legitimate Deck Problem is in $q$-LWPP.

Proof. Suppose that the $q$-Reconstruction Conjecture holds. Let $\left\langle G_{1}, G_{2}, \ldots, G_{n}\right\rangle$ be an input to the Legitimate Deck Problem. By our assumption, we have that

$$
\begin{aligned}
& \left\langle G_{1}, G_{2}, \ldots, G_{n}\right\rangle \in \text { Legitimate Deck } \Longrightarrow \operatorname{PCount}\left(\left\langle G_{1}, G_{2}, \ldots, G_{n}\right\rangle\right) \in\{1,2, \ldots, q(n)\}, \\
& \quad \text { and } \\
& \left\langle G_{1}, G_{2}, \ldots, G_{n}\right\rangle \notin \text { Legitimate Deck } \Longrightarrow \operatorname{PCount}\left(\left\langle G_{1}, G_{2}, \ldots, G_{n}\right\rangle\right)=0 .
\end{aligned}
$$

Let $h \in \mathrm{FP}$ be the function discussed in Theorem 4.2. Then the function $g$ defined such that for every sequence $\left\langle G_{1}, G_{2}, \ldots, G_{n}\right\rangle$,

$$
g\left(\left\langle G_{1}, G_{2}, \ldots, G_{n}\right\rangle\right)=h\left(0^{n}\right) \cdot \operatorname{PCount}\left(\left\langle G_{1}, G_{2}, \ldots, G_{n}\right\rangle\right)
$$

is in GapP. It follows that

$$
\begin{aligned}
& \left\langle G_{1}, G_{2}, \ldots, G_{n}\right\rangle \in \text { Legitimate Deck } \Longrightarrow g\left(\left\langle G_{1}, G_{2}, \ldots, G_{n}\right\rangle\right)=h\left(0^{n}\right) \cdot i \text { for some } \\
& i \in\{1,2, \ldots, q(n)\} \text {, and } \\
& \left\langle G_{1}, G_{2}, \ldots, G_{n}\right\rangle \notin \text { Legitimate Deck } \Longrightarrow g\left(\left\langle G_{1}, G_{2}, \ldots, G_{n}\right\rangle\right)=0 \text {. }
\end{aligned}
$$

This almost directly implies that Legitimate Deck $\in q$ - $\widehat{\mathrm{LWPP}}$. However, note that to satisfy Definition 4.6, function $h$ must be a function that depends only on the length of the input 
$\left\langle G_{1}, G_{2}, \ldots, G_{n}\right\rangle$. The problem here is that (depending on how exactly we decide to encode the graphs $G_{1}, G_{2}, \ldots, G_{n}$ in the input $\left.\left\langle G_{1}, G_{2}, \ldots, G_{n}\right\rangle\right)$ even the value $n$ (the number of graphs in the deck) may depend on the actual input $\left\langle G_{1}, G_{2}, \ldots, G_{n}\right\rangle$ and not only on the length of the input $\left\langle G_{1}, G_{2}, \ldots, G_{n}\right\rangle$.

Fortunately, there is a way to get around this problem. From the length of the input, we get at least an upper bound for $n$ since we can certainly assume that $n \leq\left|\left\langle G_{1}, G_{2}, \ldots, G_{n}\right\rangle\right|$. Define a function $\widehat{h}$ such that for each $m \in \mathbb{N}, \widehat{h}\left(0^{m}\right)$ is the product of all "h-values" up to length $m$. That is, define function $\widehat{h}$ such that for all $m \in \mathbb{N}$,

$$
\widehat{h}\left(0^{m}\right)=\prod_{0 \leq i \leq m} h\left(0^{i}\right)
$$

Define function $h^{\prime}$ such that for all inputs $\left\langle G_{1}, G_{2}, \ldots, G_{n}\right\rangle$,

$$
h^{\prime}\left(\left\langle G_{1}, G_{2}, \ldots, G_{n}\right\rangle\right)=\prod_{0 \leq i \leq\left|\left\langle G_{1}, G_{2}, \ldots, G_{n}\right\rangle\right| \wedge i \neq n} h\left(0^{i}\right) .
$$

Now we can see that for all inputs $\left\langle G_{1}, G_{2}, \ldots, G_{n}\right\rangle$,

$$
\begin{aligned}
& g\left(\left\langle G_{1}, G_{2}, \ldots, G_{n}\right\rangle\right) \cdot h^{\prime}\left(\left\langle G_{1}, G_{2}, \ldots, G_{n}\right\rangle\right) \\
& \quad= \begin{cases}0 & \text { if }\left\langle G_{1}, G_{2}, \ldots, G_{n}\right\rangle \notin \\
i \cdot \widehat{h}\left(0^{\left|\left\langle G_{1}, G_{2}, \ldots, G_{n}\right\rangle\right|}\right) \text { for some } i \in\{1,2, \ldots, q(n)\} & \text { Legitimate Deck } \\
& \text { if }\left\langle G_{1}, G_{2}, \ldots, G_{n}\right\rangle \in\end{cases}
\end{aligned}
$$

Note that $\widehat{h}, h^{\prime} \in \mathrm{FP}$ and $g \in \mathrm{GapP}$. By Closure Properties 3.2 and 3.5, the function $x \mapsto$ $g(x) \cdot h^{\prime}(x)$ is in GapP. Finally, note that since $q$ is nondecreasing and $n \leq\left|\left\langle G_{1}, G_{2}, \ldots, G_{n}\right\rangle\right|$, we have that $q(n) \leq q\left(\left|\left\langle G_{1}, G_{2}, \ldots, G_{n}\right\rangle\right|\right)$ in Eqn. (3). Thus by Definition 4.6 - with the GapP function $g$ there being our $g(x) \cdot h^{\prime}(x)$ and the function $f$ there being our $\widehat{h}$ - we have that Legitimate Deck $\in q$ - $\widehat{\mathrm{LWPP}}$.

We want to show that if there exists a polynomial $q$ such that the $q$-Reconstruction Conjecture holds, then Legitimate Deck is in LWPP. To this end, we need the following inclusion.

Theorem 4.8. For each function $r: \mathbb{N} \rightarrow \mathbb{N}, r-\widehat{\mathrm{LWPP}} \subseteq r$-LWPP.

Proof. Let $A$ be a set in $r$ - $\widehat{\mathrm{LWPP}}$ via $f_{1} \in \mathrm{FP}$ and $g \in \mathrm{GapP}$. Let $f_{2} \in \mathrm{FP}$ be the function defined such that for every $n, i \in \mathbb{N}^{+}, f_{2}\left(\left\langle 0^{n}, i\right\rangle\right)=i \cdot f_{1}\left(0^{n}\right)$. Then $A$ is in $r$-LWPP via $f_{2} \in \mathrm{FP}$ and $g \in \mathrm{GapP}$.

- Corollary 4.9. If the q-Reconstruction Conjecture holds for some polynomial $q$, then the Legitimate Deck Problem is in LWPP.

Proof. Suppose the $q$-Reconstruction Conjecture holds for nondecreasing polynomial $q$. (If $q$ is not nondecreasing but the $q$-Reconstruction Conjecture holds, then obviously we can replace $q$ with a nondecreasing polynomial $q^{\prime}$ that on each input $n$ is greater than or equal to $q(n)$ and the $q^{\prime}$-Reconstruction Conjecture will hold. So we may w.l.o.g. take it that $q$ is nondecreasing.) By Theorems 4.7 and 4.8, Legitimate Deck $\in q$ - $\widehat{\mathrm{LWPP}} \subseteq q$-LWPP and hence Legitimate Deck $\in$ Poly-LWPP. With Theorem 3.1, it follows that Legitimate Deck $\in$ LWPP. 
This gives us our new, more flexible - though still conditional - evidence that the Legitimate Deck Problem is not NP-hard.

- Corollary 4.10. If the q-Reconstruction Conjecture holds for some polynomial $q$, then the Legitimate Deck Problem is not NP-hard (or even NP-Turing-hard) unless NP is PP-low.

- Definition 4.11 ([17]). Let $\mathcal{H}$ be any class of graphs. Then the Legitimate Deck Problem restricted to $\mathcal{H}$ consists of all sequences of graphs $\left\langle G_{1}, G_{2}, \ldots, G_{n}\right\rangle$ such that $\left\langle G_{1}, G_{2}, \ldots, G_{n}\right\rangle$ is a legitimate deck and for each $i \in\{1,2, \ldots, n\}, G_{i}$ is in $\mathcal{H}$.

Note that the above definition is so flexible that it allows even the case where the preimage(s) may not be in $\mathcal{H}$.

- Theorem 4.12. Let $\mathcal{H}$ be any P-recognizable class of graphs such that decks consisting only of graphs in $\mathcal{H}$ have a number of nonisomorphic preimages that is bounded polynomially in the number of graphs in the deck. Then the Legitimate Deck Problem restricted to $\mathcal{H}$ is in LWPP.

Proof. Let $\mathcal{H}$ be any P-recognizable class of graphs and $q$ a nondecreasing polynomial such that decks consisting only of graphs in $\mathcal{H}$ have a number of nonisomorphic preimages that is bounded by $q(n)$, where $n$ is the number of graphs in the deck.

First, we show that the Legitimate Deck Problem restricted to $\mathcal{H}$ is in $q$ - $\widehat{\mathrm{LWPP}}$. Let $\left\langle G_{1}, G_{2}, \ldots, G_{n}\right\rangle$ be an input to the Legitimate Deck Problem. Check if for every $i \in$ $\{1,2, \ldots, n\}, G_{i}$ is in $\mathcal{H}$. If this is not the case then reject in the sense of $q$-LWPP, i.e., produce a gap of zero. Otherwise, the deck $\left\langle G_{1}, G_{2}, \ldots, G_{n}\right\rangle$ has at most $q(n)$ preimages, i.e., PCount $\left(\left\langle G_{1}, G_{2}, \ldots, G_{n}\right\rangle \leq q(n)\right.$. Proceed as in the proof of Theorem 4.7.

Since by Theorems 4.8 and 3.1, $q$ - $\widehat{\mathrm{LWPP}} \subseteq q$-LWPP $\subseteq$ LWPP, it follows that the Legitimate Deck Problem restricted to $\mathcal{H}$ is in LWPP.

As usual, for each graph $G, \delta(G)$ denotes the degree of a minimum-degree vertex of $G$.

Theorem 4.13. For each $k \in \mathbb{N}^{+}$, let

$$
\mathcal{H}_{k}=\{G \mid G \text { is a graph such that } \delta(G) \leq k\}
$$

Then the Legitimate Deck Problem restricted to $\mathcal{H}_{k}$ is in LWPP.

Proof. In the proof of their Theorem 6.1, Kratsch and Hemaspaandra [17] showed that for each class of graphs with bounded minimum degree, the number of nonisomorphic preimages is polynomially bounded. Now the theorem follows from Theorem 4.12.

It is interesting to note that for each of the $\mathcal{H}_{k}$ classes $\mathrm{GI}_{\mathcal{H}_{k}} \equiv_{m}^{p}$ GI trivially holds (for example, via adding to each of the two graphs being tested for isomorphism an isolated node), notwithstanding the fact that intersection with $\mathcal{H}_{k}$ pulls the Legitimate Deck Problem's complexity into LWPP.

In two of this section's corollaries we used the fact that all LWPP sets are PP-low. We mention that since all LWPP set are also $\mathrm{C}_{=} \mathrm{P}-\mathrm{low}[15,8]$, the altered versions of Corollaries 4.4 and 4.10 in which the conclusion is changed from "unless NP is PP-low" to "unless NP is $\mathrm{C}_{=} \mathrm{P}$-low" both hold, respectively due to Köbler, Schöning, and Torán [15] and the present paper. 


\section{Optimality of the Main Result (Brief Version)}

It is easy to see that the proof of Theorem 3.1 breaks down if Poly-LWPP is replaced by the analogous class where the size of the set of allowed gap values can be larger than polynomial in the input length. This does not necessarily imply that the corresponding theorem does not hold. However, in this section, we establish that relativizable proof techniques are not sufficient to improve Theorem 3.1 from Poly-LWPP to $r$-LWPP for any function $r$ that is not polynomially bounded. That is, we show that our main result is optimal with respect to what can be proven by relativizable proof techniques.

- Theorem 5.1. Let $r$ be any function from $\mathbb{N}$ to $\mathbb{N}$ such that for every $c \in \mathbb{N}, r \notin \mathcal{O}\left(n^{c}\right)$. Then there exists an oracle $\mathcal{O}$ such that $r$-LWPP ${ }^{\mathcal{O}} \nsubseteq \mathrm{LWPP}^{\mathcal{O}}$.

To prove Theorem 5.1, we will encode nondeterministic oracle Turing machines by lowdegree multivariate polynomials. This technique is apparently folklore and has been used, for example, by de Graaf and Valiant [6] to construct a relativized world where the quantum complexity class EQP is not contained in the modularity-based complexity class $\mathrm{MOD}_{p^{k}} \mathrm{P}$. The general technique of replacing oracle machines by simpler combinatorial objects such as circuits, decision trees, or polynomials and then using properties of such combinatorial objects to show the existence of a desired oracle dates to the seminal work of Furst, Saxe, and Sipser [9], who made the connection between circuit lower bounds and the relativization of the polynomial hierarchy - a connection that has led to the resolution of many previously long-open relativized questions, such as the achievement of an oracle making the polynomial hierarchy infinite $[31,10]$ and of oracles making the polynomial hierarchy extend exactly $k$ levels [14].

Note: Please see [12] for a complete version of this section, which includes the proof of Theorem 5.1 and additional results showing that LWPP with an exponential number of target gap values cannot equal LWPP without causing highly unlikely structural consequences such as $\mathrm{PP}^{\mathrm{NP}}=\mathrm{PP}$.

\section{$6 \quad \mathrm{LWPP}^{+}$(Brief Version)}

Theorem 3.1 of Section 3 established a robustness property of LWPP, namely, that Poly$\mathrm{LWPP}=\mathrm{LWPP}$. That is, having one target value for acceptance and having a list of target values for acceptance yield the same class of languages, in the content of LWPP, which, recall, is defined in terms of the values of GapP functions. That robustness result is itself robust in the sense that it holds both in the real world and, it is easy to see, in every relativized world.

On the other hand, this equivalence for LWPP, in terms of descriptive richness, between one target value and a polynomial number of values, may not hold even for quite similar counting-class situations. In particular, in this section we prove that in some relativized worlds, for the analog (which we will call $\mathrm{LWPP}^{+}$) of LWPP defined in terms of \#P rather than GapP functions (following Cox and Pay [5], who recently introduced the \#P-based analogue of WPP), having even two target values for acceptance (the class Two-LWPP ${ }^{+}$) yields a richer class of languages than having one value. So it is not the case that single targets and lists of targets inherently function identically as to descriptive richness for counting classes.

- Theorem 6.1. There exists an oracle $\mathcal{O}$ such that $\left(\mathrm{LWPP}^{+}\right)^{\mathcal{O}} \subsetneq\left(\text { Two-LWPP }{ }^{+}\right)^{\mathcal{O}}$.

Note: Please see [12] for a complete version of this section, which includes definitions and the proof of our relativized result (Theorem 6.1) showing that, for the \#P-based analogue 
of LWPP, even two target values may yield a larger class of languages than does one target value, in contrast to what holds for the LWPP case.

\section{Conclusions and Open Questions}

In this paper, we proved that LWPP and WPP are robust enough that they remain unchanged when their single target gap is allowed to be expanded to polynomial-sized lists. We then applied this new robustness of LWPP to show that the PP-lowness of the Legitimate Deck Problem follows from a weaker hypothesis than was previously known. In doing so, we provided enhanced evidence that the Legitimate Deck Problem is not NP-hard or even NPTuring-hard. We also showed: that the polynomial-target robustness of LWPP that we have established is optimal (i.e., cannot be extended to any superpolynomial number of targets) with respect to relativizable proofs; that for the \#P-based analogue of the (GapP-based) class LWPP, in some relativized worlds even two targets give more languages than one target; that our robustness of LWPP holds even when one simultaneously expands both the acceptance target-gap set and the rejection target-gap set to be polynomial-sized lists; and that our main results also hold for WPP.

Regarding the Reconstruction Conjecture, we proved as a consequence of our results that if there exists a polynomial $q$ such that the $q$-Reconstruction Conjecture holds, then the Legitimate Deck Problem is both PP-low and $\mathrm{C}_{=} \mathrm{P}$-low. Since NP is widely believed not to be PP-low or $\mathrm{C}_{=} \mathrm{P}$-low, this provides strengthened evidence that the Legitimate Deck Problem is not NP-hard or even NP-Turing hard (since otherwise even the "Poly"-Reconstruction Conjecture must fail, yet even the far stronger Reconstruction Conjecture is generally believed to hold).

A natural open problem is whether the Legitimate Deck Problem is $\Sigma_{k}^{p}$-low [24] for some $k$, i.e., whether for some $k$ it holds that $\left(\Sigma_{k}^{p}\right)^{\text {Legitimate Deck }}=\Sigma_{k}^{p}$. Proving that that holds - though we mention that this has been a known open issue for more than two decades (see [17]) - would imply that the Legitimate Deck Problem cannot be NP-complete (even with respect to more flexible reductions such as Turing reductions and strong nondeterministic reductions [19]) unless the polynomial hierarchy collapses.

\section{References}

1 J. Bondy. A graph reconstructor's manual. In Surveys in Combinatorics, London Mathematical Society Lecture Notes Series 66, pages 221-252. Cambridge University Press, 1991.

2 J. Bondy and R. Hemminger. Graph reconstruction - a survey. Journal of Graph Theory, 1:227-268, 1977.

3 D. Bovet, P. Crescenzi, and R. Silvestri. A uniform approach to define complexity classes. Theoretical Computer Science, 104(2):263-283, 1992.

4 J. Cai, T. Gundermann, J. Hartmanis, L. Hemachandra, V. Sewelson, K. Wagner, and G. Wechsung. The boolean hierarchy II: Applications. SIAM Journal on Computing, 18(1):95-111, 1989.

5 J. Cox and T. Pay. An overview of some semantic and syntactic complexity classes. Technical Report arXiv:1806.03501 [cs.CC], ArXiv.org, June 2018.

6 M. de Graaf and P. Valiant. Comparing EQP and $\mathrm{MOD}_{p^{k}} \mathrm{P}$ using polynomial degree lower bounds. Technical Report quant-ph/0211179, Quantum Physics, 2002.

7 The Editors (of the Journal of Graph Theory). Editorial note. Journal of Graph Theory, 1(3), 1977.

8 S. Fenner, L. Fortnow, and S. Kurtz. Gap-definable counting classes. Journal of Computer and System Sciences, 48(1):116-148, 1994. 
9 M. Furst, J. Saxe, and M. Sipser. Parity, circuits, and the polynomial-time hierarchy. Mathematical Systems Theory, 17:13-27, 1984.

10 J. Håstad. Computational Limitations of Small-Depth Circuits. MIT Press, 1987.

11 E. Hemaspaandra, L. Hemaspaandra, S. Radziszowski, and R. Tripathi. Complexity results in graph reconstruction. Discrete Applied Mathematics, 155(2):103-118, 2007.

12 E. Hemaspaandra, L. Hemaspaandra, H. Spakowski, and O. Watanabe. The robustness of LWPP and WPP, with an application to graph reconstruction. Technical Report arXiv:1711.01250v2 [cs.CC], ArXiv.org, November 2017. Revised, April 2018.

13 P. Kelly. On Isometric Transformations. PhD thesis, University of Wisconsin, USA, 1942.

14 K. Ko. Relativized polynomial-time hierarchies having exactly $k$ levels. SIAM Journal on Computing, 18(2):392-408, 1989.

15 J. Köbler, U. Schöning, and J. Torán. Graph isomorphism is low for PP. Computational Complexity, 2:301-330, 1992.

16 D. Kratsch and L. Hemachandra. On the complexity of graph reconstruction. In Proceedings of the 8th Conference on Fundamentals of Computation Theory, pages 318-328. SpringerVerlag Lecture Notes in Computer Science \#529, 1991.

17 D. Kratsch and L. Hemaspaandra. On the complexity of graph reconstruction. Mathematical Systems Theory, 27(3):257-273, 1994.

18 J. Lauri and R. Scapellato. Topics in Graph Automorphisms and Reconstruction. Cambridge University Press, 2003.

19 T. Long. Strong nondeterministic polynomial-time reducibilities. Theoretical Computer Science, 21:1-25, 1982.

20 A. Mansfield. The relationship between the computational complexities of the legitimate deck and isomorphism problems. Quart. J. Math. Ser., 33(2):345-347, 1982.

21 B. Manvel. Reconstruction of graphs: Progress and prospects. Congressus Numerantium, 63:177-187, 1988.

22 C. St. J. A. Nash-Williams. The reconstruction problem. In L. Beineke and R. Wilson, editors, Selected Topics in Graph Theory, pages 205-236. Academic Press, 1978.

23 M. Ogiwara and L. Hemachandra. A complexity theory for feasible closure properties. Journal of Computer and System Sciences, 46(3):295-325, 1993.

24 U. Schöning. A low and a high hierarchy within NP. Journal of Computer and System Sciences, 27:14-28, 1983.

25 J. Simon. On Some Central Problems in Computational Complexity. PhD thesis, Cornell University, Ithaca, N.Y., 1975. Available as Cornell Department of Computer Science Technical Report TR75-224.

26 H. Spakowski, M. Thakur, and R. Tripathi. Quantum and classical complexity classes: Separations, collapses, and closure properties. Information and Computation, 200(1):1-34, 2005 .

27 L. Stockmeyer and A. Meyer. Word problems requiring exponential time. In Proceedings of the 5th ACM Symposium on Theory of Computing, pages 1-9. ACM Press, 1973.

28 S. Ulam. A Collection of Mathematical Problems. Interscience Publishers, New York, 1960.

29 K. Wagner. The complexity of combinatorial problems with succinct input representations. Acta Informatica, 23(3):325-356, 1986.

30 C. Wrathall. Complete sets and the polynomial-time hierarchy. Theoretical Computer Science, 3:23-33, 1977.

31 A. Yao. Separating the polynomial-time hierarchy by oracles. In Proceedings of the 26th IEEE Symposium on Foundations of Computer Science, pages 1-10, 1985. 